\title{
Nonexistence of a Weakly Neighbourly Polyhedral Map of Type $\{6,6\}$
}

\author{
Nandini Nilakantan
}

\section{CONTENTS}

1. Introduction

2. Proofs

Acknowledgments

References

2000 AMS Subject Classification: Primary 52B70, 51M20, 57M20

Keywords: Polyhedral maps, polyhedral 2-manifolds, regular graph design
For the existence of an $n$-vertex polyhedral map of type $\{p, p\}$, it is known that $n$ must be $\geq(p-1)^{2}$ and equality holds if and only if $K$ is weakly neighbourly. We have seen in [Brehm et al. 02] that there is a unique polyhedral map of type $\{5,5\}$ on 16 vertices. In [Brehm 90], Brehm constructed a polyhedral map of type $\{6,6\}$ with 26 vertices. In this article, we prove that there do not exist any polyhedral maps of type $\{6,6\}$ on 25 vertices. As a consequence, we show that the minimum number of edges in polyhedral maps of Euler characteristic -25 is $>75$.

\section{INTRODUCTION}

A finite collection $K$ of cycles, edges, and vertices of a complete graph is called a polyhedral complex (of dimension 2) if (i) each edge of a cycle in $K$ is in $K$, (ii) each vertex of each edge in $K$ is in $K$, and (iii) any two cycles have at most one common edge. If $u$ and $v$ are vertices of a face $F$ and $u v$ is not an edge of $F$, then $u v$ is called a $d i$ agonal. We denote a face $u_{1} \cdots u_{m} u_{1}$ either by $u_{1} \cdots u_{m}$ or $\left(u_{1}, \ldots, u_{m}\right)$ and a diagonal (or edge) $u v$ by $(u, v)$.

The vertex-set of a complex $K$ is denoted by $V(K)$. For a complex $K$, the edge graph $\operatorname{EG}(K)$ of $K$ is the graph whose vertices and edges are the vertices and edges of $K$, respectively.

A polyhedral complex $K$ is called a polyhedral 2manifold if $\mathrm{EG}(K)$ is connected and for each vertex $v$ of $K$, the faces containing $v$ are of the form $\left(v, u_{1}, \ldots, u_{2}\right), \ldots,\left(v, u_{m-1}, \ldots, u_{m}\right),\left(v, u_{m}, \ldots, u_{1}\right)$ for some $m \geq 3$.

A polyhedral 2-manifold $K$ defines a decomposition of a 2-manifold $M$ into abstract polygonal discs whose interiors are open discs which are pairwise distinct. Such a 2-manifold $M$ is unique up to homeomorphism and is called the geometric carrier of $K$.

A polyhedral 2-manifold is called a polyhedral map if the intersection of any two faces is empty, a vertex, or

(c) A K Peters, Ltd. $1058-6458 / 2003 \$ 0.50$ per page Experimental Mathematics 12:3, page 257 
an edge. Hence, a diagonal in a polyhedral map is the diagonal of a unique face and is not an edge.

If $f_{0}(K), f_{1}(K)$, and $f_{2}(K)$ are the number of vertices, edges, and faces of a complex $K$, then $\chi(K):=f_{0}(K)-$ $f_{1}(K)+f_{2}(K)$ is called the Euler characteristic of $K$.

If the number of diagonals in a polyhedral map $K$ on $n$ vertices is $d(K)$, then it is clear that $d(K)+f_{1}(K) \leq$ $\left(\begin{array}{l}n \\ 2\end{array}\right)$. A polyhedral map is called a weakly neighbourly polyhedral map (in short, wnp map) if any pair of vertices is in a face. So, an $n$-vertex polyhedral map $K$ is weakly neighbourly if and only if $d(K)+f_{1}(K)=\left(\begin{array}{l}n \\ 2\end{array}\right)$.

A polyhedral 2-manifold $K$ is said to be a polyhedral 2-manifold of type $\{p, q\}$ or a $\{p, q\}$ equivelar polyhedral 2-manifold, if each face is a $p$-cycle and the degree of each vertex is $q$ (see [Brehm and Schulte 97, Brehm and Wills 93, Datta and Nilakantan 01]).

If $K$ is a polyhedral map of type $\{p, q\}$ on $n$ vertices, then $d(K)=n q(p-3) / 2$ and $f_{1}(K)=n q / 2$. Here, we are interested in those cases where $p=q$. For a polyhedral map $K$ of type $\{p, p\}$, we see that $n p(p-3) / 2+n p / 2 \leq$ $n(n-1) / 2$ and therefore $n \geq(p-1)^{2}$. Thus, if $K$ is a wnp map of type $\{p, p\}$, then $n=(p-1)^{2}$. Clearly, the 4 -vertex 2 -sphere is the unique wnp map of type $\{3,3\}$. In [Brehm 90], Brehm proved that there exist exactly three wnp maps of type $\{4,4\}$ (the geometric carrier of two of these is the torus and that of the third is the Klein bottle). In [Brehm 90], Brehm constructed the following:

Example 1.1. Two maps:

$$
\begin{aligned}
& F=((i, i+1, i+2, i+5, i+8): 1 \leq i \leq 16\} . \\
& \text { (Additions are modulo 16.) } \\
& S=\quad\{(i, i+1, i+2, i+5, i+8, i+17): 1 \leq i \leq 26\} .
\end{aligned}
$$

(Additions are modulo 26.)

$F$ is a polyhedral map of type $\{5,5\}$ and $S$ is a polyhedral map of type $\{6,6\} . F$ is weakly neighbourly, but $S$ is not (e.g., vertices 1 and 14 are not in any common face). The geometric carriers of $F$ and $S$ are the orientable surface of Euler characteristic -8 and the nonorientable surface of Euler characteristic -26 , respectively.

For the existence of an $n$-vertex polyhedral map $M$ of type $\{6,6\}$, it is obvious that $n$ is $\geq 7$. Here, we present such a polyhedral 2-manifold with 12 vertices.

Example 1.2. A polyhedral 2-manifold of type $\{6,6\}$ with 12 vertices:

$$
P=\left\{u_{i} u_{i+1} u_{i+2} u_{i+4} u_{i+6} u_{i+9}: 1 \leq i \leq 12\right\} .
$$

(Additions are modulo 12.)
Clearly, the number of edges in $P$ is 36 and hence $\chi(P)=$ -12 . It is not difficult to see that the geometric carrier of $P$ is a nonorientable surface.

In [Brehm et al. 02], we have shown that $F$ is the unique 16-vertex polyhedral map of type $\{5,5\}$. Here we prove:

Theorem 1.3. There does not exist any polyhedral map of type $\{6,6\}$ on 25 vertices.

For $\chi \leq 1$, let $E(\chi)$ be the smallest number $E$ for which there exists a polyhedral map of Euler characteristic $\chi$ with $E$ edges. From a result (Proposition 2 in [Brehm 90]), it is seen that if $M$ is a polyhedral map of Euler characteristic -25 , then the number of edges of $M$ is $\geq 75$. So, $E(-25) \geq 75$. Here we prove:

Corollary 1.4. $E(-25)>75$.

Remark 1.5. For an $n$-vertex polyhedral map $K$ of type $\{p, p\}$, the following are equivalent: (i) $K$ is weakly neighbourly, (ii) $n=(p-1)^{2}$, and (iii) $\chi(K)=(p-1)^{2}(4-p) / 2$. So, we can replace the assumption "weakly neighbourly" by " 25 -vertex" or by "of Euler characteristic -25 " in the theorem.

Remark 1.6. From the theorem, it follows that for the existence of an $n$-vertex polyhedral map of type $\{6,6\}, n$ is $\geq 26$, where as a polyhedral 2 -manifold of type $\{6,6\}$ exists on 12 vertices (namely, $P$ in Example 1.2).

Remark 1.7. In design theory, a regular graph design (RGD) with parameters $b, v, r, k, \lambda_{1}, \lambda_{2}$ (where $\lambda_{1}-\lambda_{2}=$ \pm 1 ) is an incidence system with $v$ points and $b$ blocks in which each point is incident with $r$ blocks, each block is incident with $k$ points, and any two distinct points are together incident with $\lambda_{1}$ or $\lambda_{2}$ blocks. The point graph of an RGD is the graph whose vertices are the points of the RGD and two vertices are adjacent if and only if the corresponding points are together incident with $\lambda_{2}$ blocks.

Now, given a wnp map of type $\{p, q\}$, one can associate an RGD with parameters $r=q, k=p, \lambda_{1}=1, \lambda_{2}=2$ by simply declaring the vertices of the map to be the points and the vertices lying on a face to form a block. The RGD thus obtained has the following special property: (P) The induced subgraph of the point graph on each block is a cycle. 
Conversely, given an RGD with $r=q, k=p, \lambda_{1}=$ $1, \lambda_{2}=2$ satisfying $(\mathrm{P})$, one can uniquely recover the wnp map of type $\{p, q\}$. However, as far as we know, RGDs with property $(\mathrm{P})$ have not been studied in the design theory literature.

\section{PROOFS}

In this section, we give proofs of the results. We first state two propositions proved by Brehm in [Brehm 90]. We need these two propositions to prove the corollary.

Proposition 2.1. If $M$ is a polyhedral map, then $f_{1}(M) \leq$ $Y(\sqrt{2 Y}+2) / 8$, where $Y=f_{0}(M)+f_{2}(M)$. Equality holds if and only if $M$ is a wnp map of type $\{k, k\}$ with $f_{0}(M)=f_{2}(M)=(k-1)^{2}$ for some $k$.

Proposition 2.2. If $M$ is a polyhedral map with Euler characteristic $\chi$, then $f_{1}(M) \geq G(\chi)-\chi$, where $G(\chi):=$ $\min \{m \in \mathbb{N}: m(\sqrt{2 m}-6) \geq-8 \chi$ and $m \geq 8\}$.

Proof of Theorem 1.3: If possible, let there exist a 25vertex polyhedral map $M$ of type $\{6,6\}$. In this case, $f_{2}(M)=f_{0}(M)=25$ and $M$ is weakly neighbourly. Thus any two faces of $M$ have a common vertex. Let the vertices of $M$ be $1, \ldots, 25$. Without loss of generality, we can assume that the faces containing the vertex 25 are $(25,24,23,22,21,20),(20,1,2,3,4,25), \quad(25,4,5,6,7,8)$, $(25,8,9,10,11,12),(25,12,13,14,15,16)$, and $(25,16,17$, $18,19,24)$. The remaining 19 faces are of the form $(24$, $\left.19, j_{1}, k_{1}, j_{2}, i_{1}\right),\left(24, i_{1}, j_{3}, k_{2}, j_{4}, i_{2}\right),\left(24, i_{2}, j_{5}, k_{3}, j_{6}, i_{3}\right)$, $\left(24, i_{3}, j_{7}, j_{8}, i_{4}, 23\right),\left(23, i_{4}, j_{9}, k_{4}, j_{10}, i_{5}\right),\left(23, i_{5}, j_{11}, k_{5}\right.$, $\left.j_{12}, i_{6}\right),\left(23, i_{6}, j_{13}, k_{6}, j_{14}, i_{7}\right),\left(23, i_{7}, j_{15}, j_{16}, i_{8}, 22\right),(22$, $\left.i_{8}, j_{17}, k_{7}, j_{18}, i_{9}\right), \quad\left(22, i_{9}, j_{19}, k_{8}, j_{20}, i_{10}\right),\left(22, i_{10}, j_{21}, k_{9}\right.$, $\left.j_{22}, i_{11}\right), \quad\left(22, i_{11}, j_{23}, j_{24}, i_{12}, 21\right), \quad\left(21, i_{12}, j_{25}, k_{10}, j_{26}\right.$, $\left.i_{13}\right),\left(21, i_{13}, j_{27}, k_{11}, j_{28}, i_{14}\right),\left(21, i_{14}, j_{29}, k_{12}, j_{30}, i_{15}\right)$, $\left(21, i_{15}, j_{31}, j_{32}, i_{16}, 20\right),\left(20, i_{16}, j_{33}, k_{13}, j_{34}, i_{17}\right),\left(20, i_{17}\right.$, $\left.j_{35}, k_{14}, j_{36}, i_{18}\right)$, and $\left(20, i_{18}, j_{37}, k_{15}, j_{38}, 1\right)$ where $i_{1}, \ldots$, $i_{18}, j_{1}, \ldots, j_{38}, k_{1}, \ldots, k_{15} \in\{1, \ldots, 19\}$.

The unknown vertices $i_{1}, \ldots, i_{18}$ denote the vertices in level $1, j_{1}, \ldots, j_{38}$ denote the vertices in level 2 , and $k_{1}, \ldots, k_{15}$ denote the vertices in level 3 (see Figure 1). These vertices have to be suitably identified in order to arrive at a feasible solution. By adopting a method similar to that in [Brehm et al. 02], it will be virtually impossible to arrive at a solution. Hence, a computer program had to be developed. The number of combinations of the vertices to be considered was immense and demanded very high computer storage and time.
The following algorithm was adopted and was implemented in $\mathrm{C}++$. The unknown vertices in level 1 are grouped as the blocks $\left\{i_{1}, i_{2}, i_{3}\right\},\left\{i_{4}, i_{5}, i_{6}, i_{7}\right\}$, $\left\{i_{8}, i_{9}, i_{10}, i_{11}\right\}, \quad\left\{i_{12}, i_{13}, i_{14}, i_{15}\right\}, \quad\left\{i_{16}, i_{17}, i_{18}\right\}$ corresponding to those vertices in the links of the vertices $24,23,22,21$, and 20, respectively. Clearly, each of the elements in each block has to be different. For each of the above sets, the possible combinations of the vertices are evaluated by checking on the initial condition matrix. This matrix is a $25 \times 25$ symmetric matrix. The $i j$-th entry is 1 , if the vertices $i$ and $j$ are joined by an edge, -1 , if $i j$ is a diagonal, and 0 if it is neither. Clearly, all the diagonal elements of the matrix are 0 and the maximum entry is 2 (as each edge can belong to exactly two triangles). With the available data, the elements of the initial condition matrix $T$ are as given below.

$T_{1,2}=T_{1,20}=1, T_{1,3}=T_{1,4}=T_{1,25}=-1$,

$T_{2,1}=T_{2,3}=1, T_{2,4}=T_{2,20}=T_{2,25}=-1$,

$T_{3,2}=T_{3,4}=1, T_{3,1}=T_{3,20}=T_{3,25}=-1$,

$T_{4,25}=2, T_{4,3}=T_{4,5}=1, T_{4,1}=T_{4,2}=T_{4,6}=T_{4,7}=$ $T_{4,8}=T_{4,20}=-1$,

$T_{5,4}=T_{5,6}=1, T_{5,7}=T_{5,8}=T_{5,25}=-1$,

$T_{6,5}=T_{6,7}=1, T_{6,4}=T_{6,8}=T_{6,25}=-1$,

$T_{7,6}=T_{7,8}=1, T_{7,4}=T_{7,5}=T_{7,25}=-1$,

$T_{8,25}=2, T_{8,7}=T_{8,9}=1, T_{8,4}=T_{8,5}=T_{8,6}=T_{8,10}=$

$T_{8,11}=T_{8,12}=-1$,

$T_{9,8}=T_{9,10}=1, T_{9,11}=T_{9,12}=T_{1,25}=-1$,

$T_{10,9}=T_{10,11}=1, T_{10,8}=T_{10,12}=T_{10,25}=-1$,

$T_{11,10}=T_{11,12}=1, T_{11,8}=T_{11,9}=T_{11,25}=-1$,

$T_{12,25}=2, T_{12,11}=T_{12,13}=1, T_{12,8}=T_{12,9}=T_{12,10}=$

$T_{12,14}=T_{12,15}=T_{12,16}=-1$,

$T_{13,12}=T_{13,14}=1, T_{13,15}=T_{13,16}=T_{13,25}=-1$,

$T_{14,13}=T_{14,15}=1, T_{14,12}=T_{14,16}=T_{14,25}=-1$,

$T_{15,14}=T_{15,16}=1, T_{15,12}=T_{15,13}=T_{15,25}=-1$,

$T_{16,25}=2, T_{16,15}=T_{16,17}=1, T_{16,12}=T_{16,13}=T_{16,14}=$

$T_{16,18}=T_{16,19}=T_{16,24}=-1$,

$T_{17,16}=T_{17,18}=1, T_{17,19}=T_{17,24}=T_{17,25}=-1$,

$T_{18,17}=T_{18,19}=1, T_{18,16}=T_{18,24}=T_{18,25}=-1$,

$T_{19,18}=T_{19,24}=1, T_{19,16}=T_{19,17}=T_{19,25}=-1$,

$T_{20,25}=2, T_{20,1}=T_{20,21}=1, T_{20,2}=T_{20,3}=T_{20,4}=$

$T_{20,22}=T_{20,23}=T_{20,24}=-1$,

$T_{21,20}=T_{21,22}=1, T_{21,23}=T_{21,24}=T_{21,25}=-1$,

$T_{22,21}=T_{22,23}=1, T_{22,20}=T_{22,24}=T_{22,25}=-1$,

$T_{23,22}=T_{23,24}=1, T_{23,20}=T_{23,21}=T_{23,25}=-1$,

$T_{24,25}=2, T_{24,19}=T_{24,23}=1, T_{24,16}=T_{24,17}=T_{24,18}=$

$T_{24,20}=T_{24,21}=T_{24,22}=-1$,

$T_{25,4}=T_{25,8}=T_{25,12}=T_{25,16}=T_{25,20}=T_{25,24}=2$,

$T_{25,1}=T_{25,2}=T_{25,3}=T_{25,5}=T_{25,6}=T_{25,7}=T_{26,9}=$

$T_{25,10}=T_{25,11}=T_{25,13}=T_{25,14}=T_{25,15}=T_{25,17}=$

$T_{25,18}=T_{26,19}=T_{25,21}=T_{25,21}=T_{25,22}=T_{25,23}=-1$. 

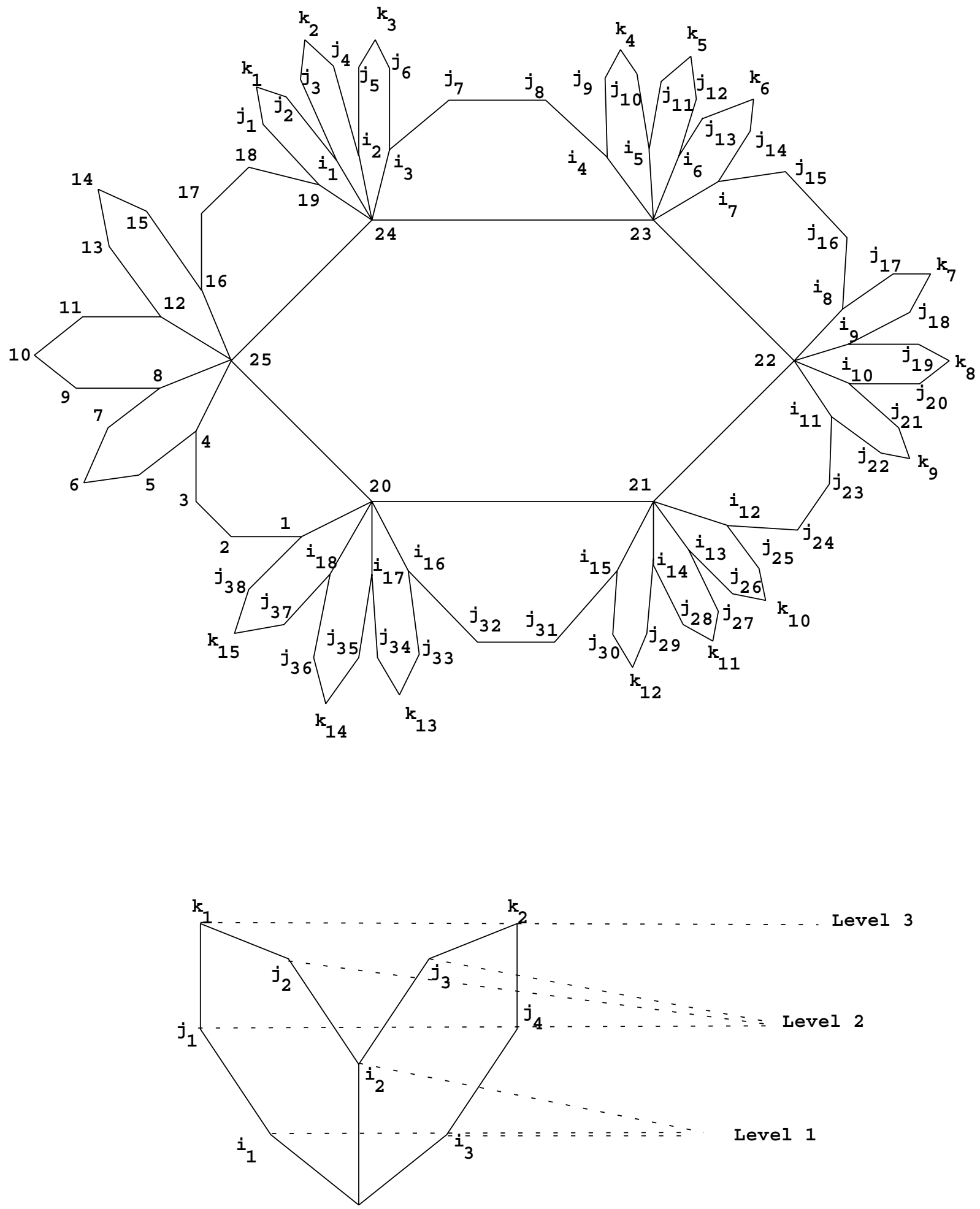

FIGURE 1. The first three levels of a possible polyhedral map in 25 vertices.

All the other entries of the initial condition matrix are 0 . For a feasible solution to exist, in each of the 25 rows, there should be exactly 6 entries which are 2 and the remaining 18 entries should be -1 (all the diagonal entries of the matrix are 0 ). At each stage in the program, the matrix is checked for the symmetry condition and for the 
existence of entries which are different from $0,1,2$, and -1 .

From the above initial condition matrix, some of the pertinent data necessary for the vertex numbering are as follows:

Number of zeros in rows 1 to 25 are $19,19,19,15$, $19,19,19,15,19,19,19,15,19,19,19,15,19,19,19$, $15,19,19,19,15$, and 0 , respectively and the number of ones in rows 1 to 25 are $2,2,2,2,2,2,2,2,2,2,2,2,2$, $2,2,2,2,2,2,2,2,2,2,2$, and 0 , respectively.

The total number of combinations possible for the sets $\left\{i_{1}, i_{2}, i_{3}\right\}, \quad\left\{i_{4}, i_{5}, i_{6}, i_{7}\right\}, \quad\left\{i_{8}, i_{9}, i_{10}, i_{11}\right\}$, $\left\{i_{12}, i_{13}, i_{14}, i_{15}\right\}$, and $\left\{i_{16}, i_{17}, i_{18}\right\}$ are 1314,39776 , 39776, 39776, and 1314, respectively. This is further reduced to $1014,22776,22776,22776$, and 1014 combinations by eliminating all those combinations where the adjacent sets do not satisfy the condition matrix. Thus, $1.0866 \times 10^{20}$ cases have been reduced to $1.2148 \times 10^{19}$ cases.

The further elimination is done as follows:

Step 1: Consider the first element in the first block.

Step 2: If $i_{1} i_{2}=i_{2} i_{3}=0$, then proceed further. Otherwise, go to Step 20.

Step 3: Consider the first element of the second block.

Step 4: : If $i_{3} i_{4}=i_{4} i_{5}=i_{5} i_{6}=i_{6} i_{7}=0$ and $i_{4} \neq i_{1}$, $i_{2}, i_{3}$ and $i_{3} \neq i_{5}, i_{6}, i_{7}$, then proceed. Otherwise, go to Step 19.

Step 5: Check for the conditions to be satisfied in the condition matrix for the different combinations of vertices in the first two blocks. If satisfied, then proceed further. Otherwise, go to Step 19.

Step 6: Consider the first element of the third block.

Step 7: If $i_{8} i_{9}=i_{9} i_{10}=i_{10} i_{11}=i_{11} i_{12}=0$ and $i_{8} \notin\left\{i_{3}, i_{4}, i_{5}, i_{6}, i_{7}\right\}, i_{7} \notin\left\{i_{9}, i_{10}, i_{11}\right\}$, then proceed. Otherwise, go to Step 18.

Step 8: Check for the conditions to be satisfied in the condition matrix for the different combinations of vertices in the first two blocks. If satisfied, then proceed further. Otherwise, go to Step 18.

Step 9: Consider the first element of the fourth block.

Step 10: If $i_{11} i_{12}=i_{12} i_{13}=i_{13} i_{14}=i_{14} i_{15}=0$ and $i_{12} \notin\left\{i_{7}, i_{8}, i_{9}, i_{10}, i_{11}\right\}, i_{11} \notin\left\{i_{13}, i_{14}, i_{15}\right\}$, then proceed. Otherwise, go to Step 17.
Step 11: Check for the conditions to be satisfied in the condition matrix for the different combinations of vertices in the first two blocks. If satisfied, then proceed further. Otherwise, go to Step 17.

Step 12: Consider the first element of the fifth block

Step 13: If $i_{15} i_{16}=i_{16} i_{17}=i_{17} i_{18}=0$ and $i_{16} \notin$ $\left\{i_{11}, i_{12}, i_{13}, i_{14}, i_{18}\right\}, i_{17} \neq i_{15}$, then proceed. Otherwise, go to Step 16.

Step 14: Check for the conditions to be satisfied in the condition matrix for the different combinations of vertices in the first two blocks. If satisfied, then proceed further. Otherwise, go to Step 16.

Step 15: If a possible solution exists in level 1 with vertices $i_{1}, i_{2}, i_{3}, i_{4}, i_{5}, i_{6}, i_{7}, i_{8}, i_{9}, i_{10}, i_{11}, i_{12}, i_{13}$, $i_{14}, i_{15}, i_{16}, i_{17}, i_{18}$, then the selection of vertices in the levels 2 and 3 are made in a very simplified way. This is performed by evaluating for a particular vertex in level 1 the following:

(i) the number of $-1 \mathrm{~s}$ in level 1 and in the adjacent levels for which this particular vertex is a common vertex.

(ii) the number of $1 \mathrm{~s}$ required for each vertex. If the sum of these is less than the total number of $0 \mathrm{~s}$ and $1 \mathrm{~s}$ available for all the vertices in level 1 , only then will a possible solution exist. Otherwise, the set arrived at is rejected.

As an example, consider the set

rej $0555431201745293-1 * 253627941028$ $371059610 * 11-1-1 * 26612665019221 *$ 21230

This corresponds to the 1st element in block 1, 5555th element in block 2, 3121th element in block 3, 1746th element in block 4, and 294th element in block 5 of level 1 . The set $\{2,5,3,6,2,7,9,4,10,2,8,3,7,10,5,9,6,10\}$ corresponds to the vertices in level 1 . In this set, the link of vertex 2 requires 6 -1s from the adjacent elements in level 1 (i.e., there have to be 6 vertices from level 1 , each of which is a diagonal with 2), $12-1$ s from the adjacent elements in the other levels, 6 additional $1 \mathrm{~s}$ and 6 vertices from the interior vertices $20,21,22,23,24,25$. This totals up to 30 . This is impossible as only 190 and 2 s are available as seen from the condition matrix. Hence, this set is rejected. If it is not, then a possible solution exists. 
This procedure is carried out for all the combinations in each block of level 1. Proceed further.

Step 16: Consider the next set of $\left\{i_{16}, i_{17}, i_{18}\right\}$ in the fifth block and go to Step 13.

Step 17: Consider the next set of $\left\{i_{12}, i_{13}, i_{14}, i_{15}\right\}$ in the fourth block and go to Step 10.

Step 18: Consider the next set of $\left\{i_{8}, i_{9}, i_{10}, i_{11}\right\}$ in the third block and go to Step 7 .

Step 19: Consider the next set of $\left\{i_{4}, i_{5}, i_{6}, i_{7}\right\}$ in the second block and go to Step 4 .

Step 20: Consider the next set of $\left\{i_{1}, i_{2}, i_{3}\right\}$ in the first block and go to Step 2.

It is observed from the computations performed that no possible solution exists to this problem. Hence, the theorem follows.

Proof of Corollary 1.4: Clearly, $G(-25)=50$. So, by Proposition $2.2, E(-25) \geq 75$. If possible, let $M$ be a polyhedral map with $\chi(M)=-25$ and $f_{1}(M)=75$. Then $f_{0}(M)+f_{2}(M)=50$. Hence, by Proposition 2.1, $M$ is a $\{k, k\}$-equivelar wnp map with $f_{0}(M)=f_{2}(M)=$ $(k-1)^{2}$ for some $k$. Then $(k-1)^{2}=25$ and hence $k=6$. The corollary now follows from the theorem.

Remark 2.3. Due to the vast number of calculations involved, we believe that the algorithm is in the most optimal form.

\section{ACKNOWLEDGMENTS}

I thank Basudeb Datta for suggesting the problem and also for many useful discussions. I thank my father and also Hariharan for helping me a lot with the programming. I thank Bhaskar Bagchi for explaining the relation between equivelar wnp maps and regular graph designs. Remark 1.7 is due to him. I thank the anonymous referee for many useful comments which have led to substantial improvements in the presentation of the paper. I am very grateful to the National Board for Higher Mathematics, DAE, India for providing me with financial support.

\section{REFERENCES}

[Brehm 90] U. Brehm. "Polyhedral Maps with Few Edges." In Topics in Comb. and Graph Theory (RingelFetstschrift), edited by R. Bodendiek and R. Henn, pp. 153-162. Heidelberg: Physica-Verlag, 1990.

[Brehm et al. 02] U. Brehm, B. Datta, and N Nilakantan. "The Edge-Minimal Polyhedral Maps of Euler Characteristic -8." Beiträge zur Algebra und Geometrie 43 (2002), 583-596.

[Brehm and Schulte 97] U. Brehm and E. Schulte. "Polyhedral Maps." In Handbooks of Discrete and Computational Geometry, edited by J. E. Goodman and J. O'Rourke, pp. 345-358. Orlando, FL: CRC Press, 1997.

[Brehm and Wills 93] U. Brehm amd J. M. Wills. "Polyhedral Manifolds." In Handbook of Convex Geometry, edited by P. M. Gruber and J. M. Wills, pp. 535-554. New York: Elsevier Publishers, 1993.

[Datta and Nilakantan 01] B. Datta and N. Nilakantan. "Equivelar Polyhedra with Few Vertices." Discrete and Comput Geom. 26 (2001), 429-461.

Nandini Nilakantan, Mathematics Section, The Abdus Salam, International Center for Theoretical Physics, Trieste, Italy (nandini@ictp.trieste.it) 